\title{
Spatiotemporal Optical Modulation Instability of Coherent Light in Noninstantaneous Nonlinear Media
}

\author{
Ming-Feng Shih,* Chien-Chung Jeng, Fan-Wen Sheu, and Chao-Yin Lin \\ Physics Department, National Taiwan University, Taipei, 106, Taiwan
}

(Received 23 August 2001; published 19 March 2002)

\begin{abstract}
We report on the theoretical derivation and experimental observation of spatiotemporal modulation instability (MI) of a coherent light beam in noninstantaneous nonlinear media. We obtain analytically the MI growth rate as a function of the spatial and temporal frequencies of the perturbation and the material response time. In the experiment, we observe that the varying speed of the MI patterns increases with the decreased material response time. We also observe that increasing the material response time can arrest the MI, agreeing with our theoretical derivation.
\end{abstract}

DOI: 10.1103/PhysRevLett.88.133902

PACS numbers: 42.65.Sf

Recent research about optical spatial solitons has made much progress [1-7], including the discoveries of new materials that can support spatial solitons and interesting phenomena about solitons. To form optical spatial solitons is simple: the natural diffraction of a light beam is exactly balanced by the self-focusing effect. It is the same competition between the diffraction and self-focusing effects that causes the modulation instability (MI) $[8,9]$. In other systems, MI has been widely studied in fluid dynamics [10] and plasma physics [11]. The formation of optical MI is similar to forming optical spatial solitons but with different initial conditions. The input light beam should be extended to a wider (infinite ideally) region rather than being localized. When the light beam carrying noise propagates in a self-focusing medium, it yields slightly higher refractive index in regions with slightly higher intensity. As the light propagates, the higher index regions attract more light nearby and yield even higher indices that attract more light. If this self-focusing effect through positive feedback is stronger than the diffraction effect, the light begins to localize. This more localized light then causes the diffraction effect to grow. When finally the diffraction is enough to balance the self-focusing, the geometry of the MI is decided and the MI patterns form. Thus MI of an incoherent light beam, which diffracts more, requires larger self-focusing nonlinearity [12]. Like incoherent solitons $[3-7,13]$, MI of incoherent light must be observed in noninstantaneous nonlinear media, in which the index change depends on the optical intensity averaged over a period longer than the material response time.

On the other hand, there is no such requirement to form MI of coherent light. If, as traditionally thought, the noise that causes the coherent MI is static (e.g., material defects), then it makes no difference whether the nonlinear media is instantaneous or noninstantaneous. The MI patterns form simply depending on the static noise distribution. Nevertheless, if the noise is dynamic (e.g., random scattering of light in air), there is a major difference between the MI that happens in instantaneous and in noninstantaneous media. In instantaneous media, MI patterns change instantly depending on the always-changing (dynamic) noise distribution when that part of the light beam just enters the media. However, in noninstantaneous media, the situation is totally different. Since the index change in noninstantaneous media is decided by the averaged optical intensity, the always-changing noise cannot alter the index dramatically at any moment, but to modify the index, to modulate the phase of the light beam, and to change the consequent MI patterns a little bit. As a result, the cumulative change of MI patterns is noticeable only after a time period comparable to the material response time.

In this Letter, we report on the theoretical analysis and experimental observation of such spatiotemporal MI of coherent light in noninstantaneous nonlinear media. We obtain the MI growth rate as a function of the noise frequency and the material response time. In the experiment, we observe that the varying speed of the MI patterns increase with the decreased material response time, indicating the noise that causes MI is indeed dynamic. We also observe that increasing the material response time can arrest the MI, agreeing with the theory.

In the analysis, starting from the wave equation with $E=A(\vec{r}, t) \exp (i \omega t-i k z)$, neglecting terms containing $\partial(\delta n) / \partial t$ and $\partial^{2}(\delta n) / \partial t^{2}\left(\ll \partial^{2} E / \partial t^{2} \approx \omega^{2}\right.$ under the noninstantaneous assumption), and utilizing slowly varying amplitude assumption, we get

$$
\nabla_{\perp}^{2} A-2 i k \frac{\partial A}{\partial z}+2 k^{2} \frac{\delta n\left(|A|^{2}\right)}{n_{0}} A=0 .
$$

In Eq. (1), $k=\sqrt{\mu \varepsilon_{0}} n_{0} \omega$ is the wave vector with $\omega$ the optical frequency, $n_{0}$ the unperturbed refractive index, $A$ the amplitude, and $\delta n$ the optical nonlinearity. Assuming the nonlinearity is relaxed with time constant $\tau$ [13] (the derivation for other types of noninstantaneity can be accomplished similarly), we express the optical nonlinearity as $\delta n\left(|E(t)|^{2}\right)=\int_{-\infty}^{t}(1 / \tau) F\left[\left|E\left(t_{1}\right)\right|^{2}\right] \exp [-(t-$ $\left.\left.t_{1}\right) / \tau\right] d t_{1}$ in which $F(I)$ is the form of the nonlinearity. For example, $F(I)=n_{2} I$ for Kerr nonlinearity and $F(I)=n_{2} I /\left(1+I / I_{\mathrm{sat}}\right)$ for saturable nonlinearity [14]. 
To study MI, we put in an unperturbed plane wave plus a perturbation, $A=\left(A_{0}+a\right) \exp (-i \gamma z)$ with $\gamma=$ $k F\left(A_{0}^{2}\right) / n_{0}$, and Eq. (1) then becomes

$$
\begin{aligned}
\nabla_{\perp}^{2} a- & 2 i k \frac{\partial a}{\partial z}-2 k \gamma\left(A_{0}+a\right)+ \\
2 & k^{2} \frac{\delta n\left(\left|A_{0}+a\right|^{2}\right)}{n_{0}}\left(A_{0}+a\right)=0 .
\end{aligned}
$$

For the perturbation eigenmode as $a=a_{1}+i a_{2}$ with $a_{1,2}=\operatorname{Re}\left\{a_{1,2}^{0} \exp \left(i \Omega \cdot t+i \vec{k}_{\perp} \cdot \vec{r}-i h z\right)\right\}$, and by the help that $F\left(\left|A_{0}+a\right|^{2}\right) \approx F\left(A_{0}^{2}\right)+2 \kappa A_{0} a_{1}$ with $\kappa=$ $[d F(I) / d I]$ being evaluated at $I=A_{0}^{2}$, and $\delta n\left(\mid A_{0}+\right.$ $\left.\left.a\right|^{2}\right) \approx F\left(A_{0}^{2}\right)+\operatorname{Re}\left\{2 A_{0} \kappa a_{1}^{0} \exp \left(i \Omega \cdot t+i \vec{k}_{\perp} \cdot \vec{r}-\right.\right.$

$i h z) /(1+i \Omega \tau)\}$ with the integration being carried out, Eq. (2) is approximated to the first order as

$$
\begin{gathered}
\nabla_{\perp}^{2}\left(a_{1}+i a_{2}\right)-2 i k \frac{\partial\left(a_{1}+i a_{2}\right)}{\partial z}+ \\
2 k^{2} \operatorname{Re}\left\{\frac{2 A_{0}^{2} \kappa a_{1}^{0}}{n_{0}(1+i \Omega \tau)} \exp \left(i \Omega t+i \vec{k}_{\perp} \cdot \vec{r}-i h z\right)\right\}=0 .
\end{gathered}
$$

In the perturbation, $\Omega$ and $\vec{k}_{\perp}$ are the temporal and spatial frequencies of the noise, respectively, and the imaginary part of $h$ is the MI growth rate. Separating the real and imaginary parts, we obtain the condition for Eq. (3) to have a nontrivial solution, that is, $h^{2}=\left(h_{1}+i h_{2}\right)^{2}=$ $k_{\perp}^{4} / 4 k^{2}-\left(\kappa / n_{0}\right) k_{\perp}^{2} A_{0}^{2} /(1+i \Omega \tau)$. Thus the MI growth rate is

$$
\begin{aligned}
h_{2}=\frac{\kappa}{n_{0}} k A_{0}^{2} \frac{1}{\sqrt{2}}[ & -P^{4}+\frac{2 P^{2}}{1+(\Omega \tau)^{2}} \\
& \left.+P^{2}\left(P^{4}+\frac{4-4 P^{2}}{1+(\Omega \tau)^{2}}\right)^{1 / 2}\right]^{1 / 2},
\end{aligned}
$$

where $P=k_{\perp} /\left(\sqrt{2 \kappa / n_{0}} k A_{0}\right)$. Figure 1 shows $h_{2}$ as a function of $P$ and $\Omega \tau$.

When $\Omega=0$, Fig. 1 (b) shows that $h_{2}$ becomes the MI growth rate for instantaneous media [8]; i.e., there is no difference for instantaneous and noninstantaneous media if the noise is static. If the noise is dynamic, the MI patterns vary in the noninstantaneous media and the varying rate should depend on the noise frequency and the response time. More importantly, if $\Omega \tau$ is large enough, two

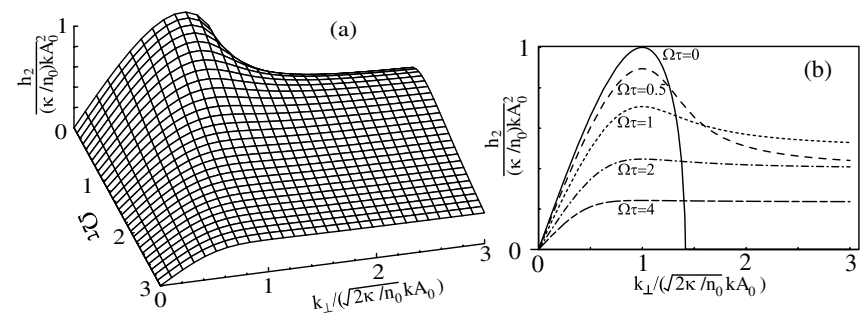

FIG. 1. The modulation instability growth rate. changes of $h_{2}$ affect MI the most: (1) $h_{2}$ is reduced, causing MI to take longer propagation to develop; and (2) $h_{2}$ is leveled off and contains no peak [Fig. 1(b) for $\Omega \tau>4$ ]. The reason MI can develop is that the noise of spatial frequency at $k_{\perp \text {,max }}$ with the peak growth rate can win over the noises of other spatial frequencies and make the refractive index environment more suitable for that particular noise of $k_{\perp \text {,max }}$ to develop into MI. When the growth rate is the same for almost all spatial frequencies, none of them is favored and the MI pattern cannot develop. In other words, the noninstantaneity plus a dynamic noise can stop MI despite the positive growth rate. This is different from the case that MI of incoherent light [12] in noninstantaneous media can be arrested by the negative growth rate when the nonlinearity is smaller than certain coherencedependent threshold.

In the experiment, we use a biased photorefractive strontium barium niobate (SBN) crystal as the noninstantaneous nonlinear medium [14], whose response time is inversely proportional to the optical intensity. We expand and collimate the extraordinarily polarized laser light beam (of $572 \mathrm{~mW} / \mathrm{cm}^{2}$ intensity and at $532 \mathrm{~nm}$ wavelength) into the SBN crystal, whose effective electro-optic coefficient $\left(r_{33}\right)$ is about $280 \mathrm{pm} / \mathrm{V}$ and refractive index about 2.35 . The light beam uniformly covers the entire crystal and propagates along the 7-mm-long crystalline $a$ axis. We use a CCD camera plus a lens to capture the image of the light beam at the output face of the crystal. When there is no voltage applied, we observe the striation pattern, a multiband structure with period about $70 \mu \mathrm{m}$, which may originate from the inhomogeneities caused by the crystal growth process. We apply a dc voltage of $0.75 \mathrm{kV}$ on the two electrodes that sandwich the 5-mm-wide crystal, with the polarity against the crystalline $c$ axis. This provides a self-focusing nonlinear index change $(\Delta n)$ with an average about $2.4 \times 10^{-4}$ for the MI to develop. The MI pattern is shown in Fig. 2(a). At the moment the voltage is just on, we observe that the movement of the MI patterns is faster, and then it slows down gradually and reaches a steady moving speed. We continue to observe for more than a few hours and never find the patterns to stop moving; i.e., the time-varying behavior of the MI pattern is not transient. (In the following experiments, we always take the images after the steady state is reached. This temporally faster movement could be related to the quasi-steady-state photorefractive solitons [15].) The movement of the patterns is shown in Fig. 2(a). For example, on the upper-left corner, a stripe (indicated by the arrowheads) disintegrates, and its position of highest intensity shifts. Other stripes of the MI pattern shown have similar movements or deformations. This obviously indicates that the noise is dynamic.

In the theory, we predict that increasing material response time can arrest MI. Therefore, we expect for a fixed strength of nonlinearity, the MI pattern can be eliminated if the response time is long enough, which can be done by reducing the optical intensity for photorefractive materials. 
(a)

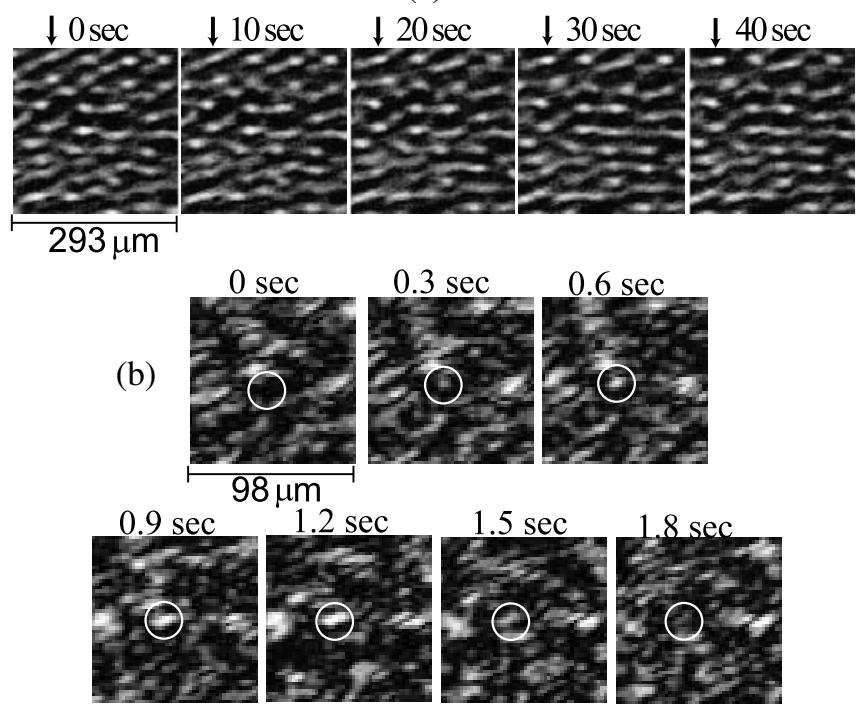

FIG. 2. (a) The movement and deformation of the MI patterns. (b) An example of the appearance and disappearance of the bright spot in the optical turbulence.

We set the voltage at $0.9 \mathrm{kV}$ and the optical intensity at different values. Figure 3(a) shows that, at $57.2 \mathrm{~mW} / \mathrm{cm}^{2}$, the MI cannot develop, and when the intensity is at or above $286 \mathrm{~mW} / \mathrm{cm}^{2}$, the MI pattern is clearly seen. Also as pointed out in the intuition, the varying speed of the MI patterns increases with the decreased response time, which is roughly inversely proportional to the optical intensity within photorefractive materials. Therefore, we fix the applied voltage, set the optical intensity to different values, and capture the images of the time-varying MI patterns with suitable time separations. The patterns are shown in Fig. 3. In each of the measurements, we observe faster varying speed when the crystal is illuminated with higher

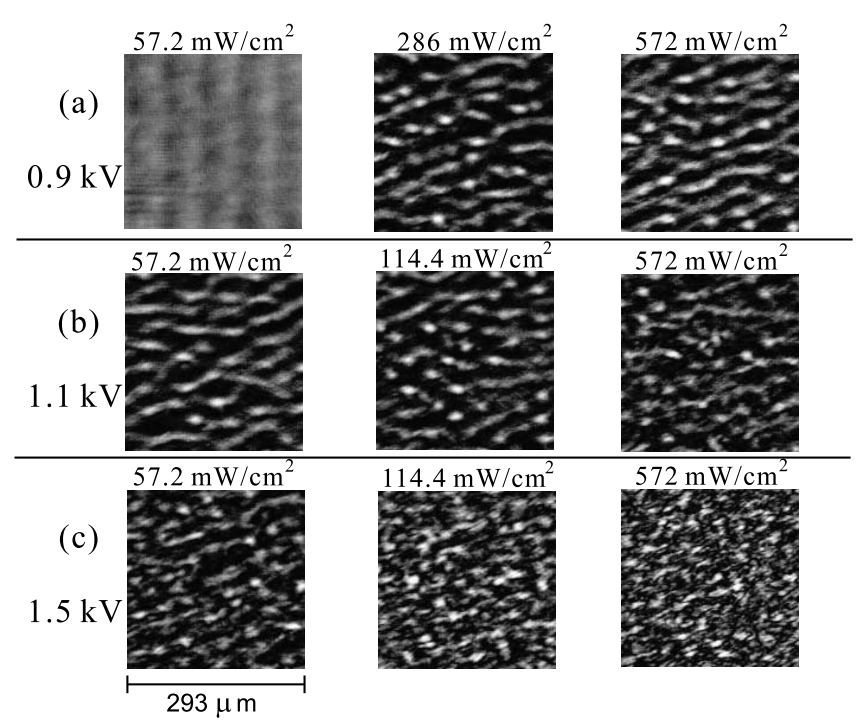

FIG. 3. MI patterns and optical turbulences formed under different incident optical intensities and applied voltages. optical intensity. Even to our surprise, we observe that the MI patterns may break up into randomly moving and randomly distributed speckles when the optical intensity is above some voltage-dependent threshold. For example, at $1.1 \mathrm{kV}$ the threshold intensity is about $572 \mathrm{~mW} / \mathrm{cm}^{2}$ and at $1.5 \mathrm{kV}$ the threshold is at about $114 \mathrm{~mW} / \mathrm{cm}^{2}$. We call these random speckles optical turbulence. We conjecture that this behavior may be related to that of the viscous flow [16]. When the optical intensity (flow rate) reaches some critical value, the MI pattern (laminar flow) undergoes a transition into optical turbulence (turbulent flow).

To characterize the varying speed of the MI patterns or of the optical turbulence, we measure the average lifetime at several randomly chosen points. The lifetime is defined as the time that the optical intensity at a specific point goes from a local minimum to a local maximum then back to a local minimum [e.g., Figs. 2(a) and 2(b)]. The trend [Fig. 4(a)] is expected: the lifetime is smaller for higher intensity (i.e., shorter response time). However, we do not observe any clear relationship between the lifetime and the threshold that MI becomes optical turbulence. Notice that, unlike a particle in a Browning motion that is traceable along its random path, whose characteristics can be identified by the mean square distance from some original point, the bright optical spots or bands here may move, disappear, appear, disintegrate, or merge together. Therefore we cannot use the mean square distance similar to that of a Browning motion to describe the time-varying MI or optical turbulence. Nevertheless, both phenomena are similar in that both are driven by the dynamically random noise.

We also set the optical intensity at constant and observe the MI patterns and optical turbulence under different applied voltages [Fig. 5]. These results are consistent with previous observation: (1) at higher intensity, the required voltage for MI to transform into optical turbulence is smaller; (2) the lifetime decreases with the increased voltage [Fig. 4(b)] since the material response time is reduced as the excited electrons in the conduction band move faster under a higher drifting field; and (3) the MI is arrested by increasing the response time [comparing the images in Fig. 5(c) for intensity at 572 and $57.2 \mathrm{~mW} / \mathrm{cm}^{2}$ ].

Before concluding, we discuss more about the differences between the instantaneous and noninstantaneous self-focusing nonlinearities. As pointed out previously, in noninstantaneous self-focusing media, the refractive
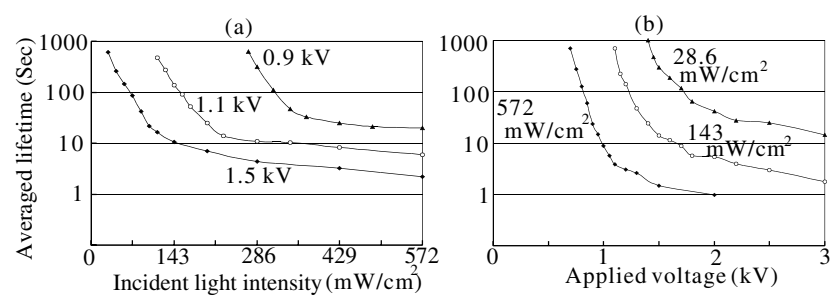

FIG. 4. The experimentally measured average lifetimes of the MI patterns or optical turbulences. 


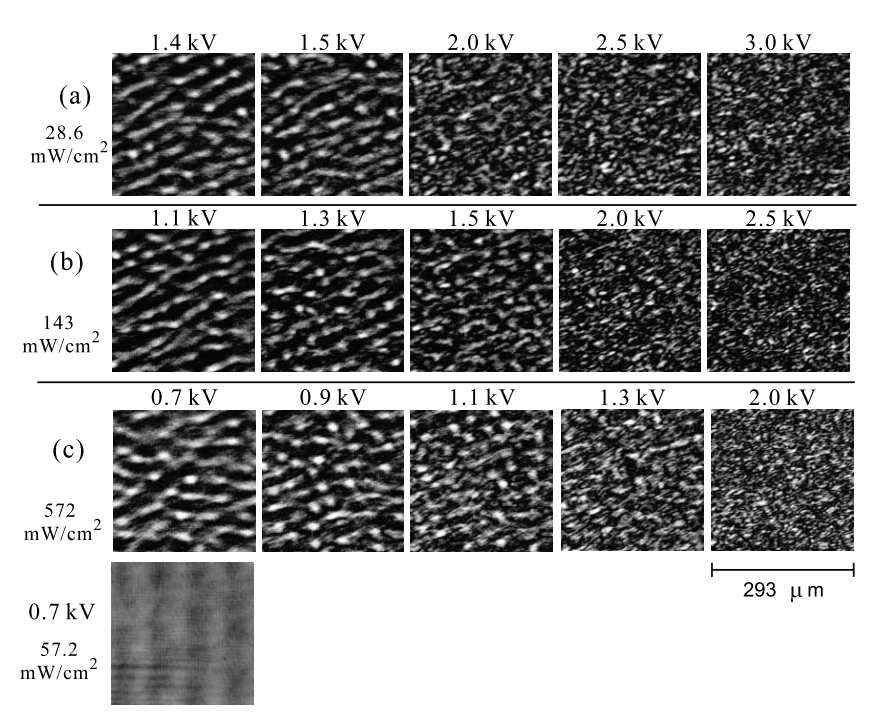

FIG. 5. MI patterns and optical turbulences formed under different applied voltages and incident optical intensities.

index cannot be altered instantly by the change of the intensity. This obviously indicates that the spatiotemporal solitons (STS) [17-20] cannot be observed in noninstantaneous self-focusing media since STS needs instant phase modulation to compensate the dispersion to prevent its very short pulse from spreading. Besides, the ability to instantly modulate the phase also affects the stability criterion of a homogeneous $\mathrm{cw}$ light beam propagating in the self-focusing medium. For example, it is shown that MI or transverse instability [20,21] is inevitable in the quadratic self-focusing medium, whose nonlinear response is instantaneous, even if a time-varying perturbation or time-varying light beam is posted. On the contrary, as we showed theoretically and experimentally here, with noninstantaneous self-focusing media, MI can be arrested by the time-varying perturbation. Nevertheless, if we set the temporal frequency of the perturbation to zero, then the curve of growth rate here and the one in Ref. [21] look very similar. Though we experiment only on the photorefractive nonlinear media, the same arguments and observations can be applied to any other noninstantaneous self-focusing media, e.g., orientational Kerr media [22], and the observation time scale should be of the same order of the response time of those materials.

In summary, spatiotemporal MI has been investigated both theoretically and experimentally in noninstantaneous nonlinear media. We find that the MI growth rate is a function of the temporal and spatial frequencies of the perturbation and the material response time. We observe that the varying speed of the MI pattern decreases with increased response time, indicating that the noise that causes the optical MI is dynamic. We also observe that increasing the material response time can arrest MI, agreeing with the theory.

This research is supported by the National Science Council, Taiwan, under Contract No. NSC-89-2112-M-002-060.

*Email address: mfshih@phys.ntu.edu.tw

[1] For a recent general review, see G. I. Stegeman and M. Segev, Science 286, 1518 (1999).

[2] M. Shih, M. Segev, and G. Salamo, Phys. Rev. Lett. 78, 2551 (1997).

[3] M. Mitchell et al., Phys. Rev. Lett. 77, 490 (1996); M. Mitchell and M. Segev, Nature (London) 387, 880 (1997); Z. Chen et al., Science 280, 889 (1998); D. N. Christodoulides et al., Phys. Rev. Lett. 80, 5113 (1998).

[4] A. W. Snyder and D. J. Mitchell, Phys. Rev. Lett. 80, 1422 (1998).

[5] V. V. Shkunov and D. Z. Anderson, Phys. Rev. Lett. 81, 2683 (1998).

[6] N. Akhmediev et al., Phys. Rev. Lett. 81, 4632 (1998).

[7] O. Bang et al., Phys. Rev. Lett. 83, 4740 (1999).

[8] V. I. Bespalov and V. I. Talanov, JETP Lett. 3, 307 (1966); V. I. Karpman, JETP Lett. 6, 277 (1967); G. P. Agrawal, Phys. Rev. Lett. 59, 880 (1987); Nonlinear Fiber Optics (Academic Press, San Diego, 1995), 2nd ed., Chap. 5.

[9] E. M. Dianov et al., Opt. Lett. 14, 1008 (1989); M. D. Iturbe-Castillo et al., Opt. Lett. 20, 1853 (1995); M. I. Carvalho et al., Opt. Commun. 126, 167 (1996).

[10] For example, G. B. Whitham, Proc. R. Soc. London A 283, 238 (1965); T. B. Benjamin and J. E. Feir, J. Fluid Mech. 27, 417 (1967).

[11] For example, T. Taniuti and H. Washimi, Phys. Rev. Lett. 21, 209 (1968); C. K. W. Tam, Phys. Fluids 12, 1028 (1969); A. Hasegawa, Phys. Rev. Lett. 24, 1165 (1970).

[12] M. Soljacic et al., Phys. Rev. Lett. 84, 467 (2000); D. Kip et al., Science 290, 495 (2000); C. Anastasia et al., Phys. Rev. Lett. 85, 4888 (2000).

[13] M. Shih and F. Sheu, Phys. Rev. Lett. 86, 2281 (2001).

[14] M. Segev et al., Phys. Rev. Lett. 73, 3211 (1994); D. N. Christodoulides and M. Carvalho, J. Opt. Soc. Am. B 12, 1628 (1995); M. Shih et al., Electron. Lett. 31, 826 (1995).

[15] M. Segev et al., Phys. Rev. Lett. 68, 923 (1992); G. Duree et al., Phys. Rev. Lett. 71, 533 (1993).

[16] L. D. Landau and E. M. Lifshitz, Fluid Mechanics (Butterworth-Heinemann, Oxford, 1997), 3rd ed., Chap. 2.

[17] Y. Silberberg, Opt. Lett. 15, 1282 (1990).

[18] X. Liu, L. J. Qian, and F. Wise, Phys. Rev. Lett. 82, 4631 (1999).

[19] I. G. Koprinkov et al., Phys. Rev. Lett. 84, 3847 (2000).

[20] X. Liu, L. Beckwitt, and F. Wise, Phys. Rev. Lett. 85, 1871 (2000).

[21] Z.H. Musslimani and B. A. Malomed, Physica (Amsterdam) 123D, 235 (1998).

[22] A. Yariv, Quantum Electronics (John Wiley \& Sons, New York, 1989), 3rd ed., Chap. 18. 